\title{
Charging of Metal Adatoms on Ultrathin Oxide Films: Au and Pd on $\mathrm{FeO} / \mathrm{Pt}(111)$
}

\author{
Livia Giordano, ${ }^{1}$ Gianfranco Pacchioni, ${ }^{1, *}$ Jacek Goniakowski, ${ }^{2}$ Niklas Nilius, ${ }^{3, *}$ \\ Emile D. L. Rienks, ${ }^{3}$ and Hans-Joachim Freund ${ }^{3}$ \\ ${ }^{1}$ Dipartimento di Scienza dei Materiali, Università di Milano-Bicocca, via Cozzi, 53-20125 Milano, Italy \\ ${ }^{2}$ Institut des Nanosciences de Paris, UMR 7588 CNRS and Université Paris 6, Campus de Boucicaut, \\ 140 Rue de Lourmel, 75015 Paris, France \\ ${ }^{3}$ Department of Chemical Physics, Fritz-Haber Institut der Max-Planck Gesellschaft, Faradayweg 4-6, D-14195 Berlin, Germany
}

(Received 23 April 2008; published 11 July 2008)

\begin{abstract}
We present a combined experimental (STM/scanning tunneling spectroscopy) and theoretical (density functional theory) study on the deposition of $\mathrm{Au}$ and $\mathrm{Pd}$ metal atoms on $\mathrm{FeO} / \mathrm{Pt}(111)$ ultrathin films. We show that while the Pd atoms are only slightly oxidized, the Au atoms form positive ions upon deposition, at variance to a charge transfer into the $\mathrm{Au}$ atoms as observed for $\mathrm{MgO} / \mathrm{Ag}(100)$. The modulation of the adsorption properties within the surface Moire cell and the charging induce the formation a self-assembled array of gold adatoms on $\mathrm{FeO} / \mathrm{Pt}(111)$, whereas $\mathrm{Pd}$ atoms are randomly distributed.
\end{abstract}

DOI: 10.1103/PhysRevLett.101.026102

Charging of metal atoms or clusters deposited on an oxide surface or on metal-supported oxide thin films represents an important physical effect which can deeply modify the chemical, optical, and magnetic properties of the supported species [1]. Recently, a new mechanism leading to a spontaneous charging of supported particles has been proposed on the basis of theoretical evidence [2$5]$ and then confirmed experimentally [6,7]. For Au atoms [2] and clusters [3] deposited on ultrathin $\mathrm{MgO}$ films supported on $\operatorname{Mo}(100)$ or $\operatorname{Ag}(100)$ substrates, electrons flow from the metal substrate to the deposited gold by direct tunneling through the oxide thin film. The process is determined by the low work function $(\Phi)$ of the $\mathrm{MgO} /$ metal substrate, the high electron affinity of the deposited atom or cluster, and the reduced thickness of the film (3-4 layers) being essential to enable tunneling. The resulting negatively charged $\mathrm{Au}$ atoms repel each other and form an ordered array, contrary to the case of neutral Pd atoms on the same substrate, which are randomly distributed [6]. Moreover, because of the charge transfer, Au clusters grow two-dimensional and completely wet the substrate on ultrathin $\mathrm{MgO}$ films, while on thicker films, they grow in three-dimensions [3,7]. A similar effect has been found on alumina films on $\mathrm{NiAl}(110)$, where deposited gold forms negatively charged chains due to the formation of direct $\mathrm{Au}-\mathrm{Al}$ bonds $[8,9]$. So far, only negative $\mathrm{Au}$ atoms and particles have been observed on metal-supported oxide films. The occurrence of positive charging has been found for alkali metals on oxide thin films [3,10,11], but in principle, an adequate tuning of the metal/oxide interface could lead also to the stabilization of transition metal atoms in a positive charged state. For instance, a metal with a large work function or an easily reducible oxide may favor the electron flow from the adatom to the substrate. In the case of polar oxide films [12-14], the orientation of the film dipole may be an additional factor which influences the direction of the charge transfer.
PACS numbers: 68.47.Gh, 68.37.Ef, 71.15.Mb, 73.63.Rt

The well characterized $\mathrm{FeO} / \mathrm{Pt}(111)$ substrate [15-19] seems to possess most of the desired characteristics to stabilize positively-charged adsorbates: a metal substrate with a high $\Phi$, a reducible oxide, and an oxide dipole moment that favors charge flow into the support. By combining STM/STS with first principles DFT calculations, we show that in this system, a net electron transfer occurs from the $\mathrm{Au}$ atom to the $\mathrm{FeO} / \mathrm{Pt}(111)$ substrate. The effect is much less pronounced for Pd atoms where only a partial oxidation is observed. The net charging combined with the modulation of the Au adsorption properties within the Moiré pattern specific to $\mathrm{FeO} / \mathrm{Pt}(111)$ produce a selfassembled array of atoms in case of Au and not of Pd. It is interesting to note that the same phenomenon, ordered versus random distribution of $\mathrm{Au}$ and $\mathrm{Pd}$ atoms, respectively, has been observed on a different system, $\mathrm{MgO} / \mathrm{Ag}(100)$ [6]. While the final effect is similar, the origin of the ordering is different, being the charge transfer into $\mathrm{Au}$ on $\mathrm{MgO} / \mathrm{Ag}(100)$ but out of the adatoms on $\mathrm{FeO} / \mathrm{Pt}(111)$.

All experiments have been conducted in an ultrahighvacuum STM operated at $5 \mathrm{~K}$. The FeO film is prepared by depositing a monolayer of $\mathrm{Fe}$ onto sputtered/annealed $\mathrm{Pt}(111)$, followed by annealing in $1 \times 10^{-6} \mathrm{mbar}_{2}$ at $950 \mathrm{~K}$. It consists of a well-ordered bi-layer of $\mathrm{FeO}(111)$, whereby the interface is formed by positively charged $\mathrm{Fe}^{\delta+}$ ions and the surface by $\mathrm{O}^{\delta-}$ ions. The film carries a macroscopic dipole moment oriented perpendicular to the surface plane. Because of the $11 \%$ lattice mismatch between $\operatorname{Pt}(111) \quad\left(d_{\mathrm{Pt}-\mathrm{Pt}}=2.76 \AA\right)$ and $\mathrm{FeO}\left(d_{\mathrm{Fe}-\mathrm{Fe}}=\right.$ $3.08 \AA$ ), the oxide lattice forms a hexagonal Moiré pattern with $25 \AA$ lattice constant on the support. Inside the Moiré unit cell, the $\mathrm{Fe}$ interface atoms adapt three different registries with respect to the Pt lattice: on top of Pt atoms (O in fcc-hollow sites), in fcc (O in hcp-hollows), and hcp hollow positions (O on top of Pt atoms), see Fig. 1(c) and Fig. 1(a) in Ref. [19]. The different stacking configurations 
give rise to changes in the $\mathrm{Fe}-\mathrm{O}$ layer distance and therefore in the strength of the surface dipole. The hcp-domains are associated to the largest surface dipole, while the top regions show the smallest dipole strength. This assignment is verified by DFT calculations on model structures of the FeO Moiré cell [19].

Single $\mathrm{Au}$ and $\mathrm{Pd}$ atoms are deposited onto $\mathrm{FeO} / \mathrm{Pt}(111)$ at $10 \mathrm{~K}$ from a resistively heated $\mathrm{W}$ filament. Because of their thermal energy when impinging the surface, the metal atoms probe a large number of adsorption sites before thermalization. The adsorption geometries revealed in the STM topographies can therefore be considered as equilibrium structures. The metal coverage is adjusted to $0.45-$ 0.75 atoms per Moiré unit cell $(\Theta<0.01 \mathrm{ML})$. Small amounts of $\mathrm{CO}$ are dosed at $5 \mathrm{~K}$ onto the surface after metal exposure.

In STM images of the FeO film taken at $0.5 \mathrm{~V}$, both $\mathrm{Pd}$ and $\mathrm{Au}$ adatoms are discernible as round protrusions of approximately $1.8 \AA$ height [Figs. 1(a) and 1(b)]. Their preferred adsorption region within the $\mathrm{FeO}$ Moiré cell (fcc, hcp, top) can be determined from the experiment, as each Fe-Pt stacking domain shows a characteristic contrast evolution with sample bias, as discussed in Refs. [18,19]. Au adatoms show a strong tendency to bind in the center of the hcp-domains, leading to the formation of a hexagonal adatom lattice with an $\mathrm{Au}-\mathrm{Au}$ distance coherent with the size of the Moiré cell [Fig. 1(a)]. On the other hand, no preferred adsorption site could be identified for the Pd adatoms.

A different long-range spatial arrangement of $\mathrm{Pd}$ and $\mathrm{Au}$ atoms on the surface is visible already in the topographic
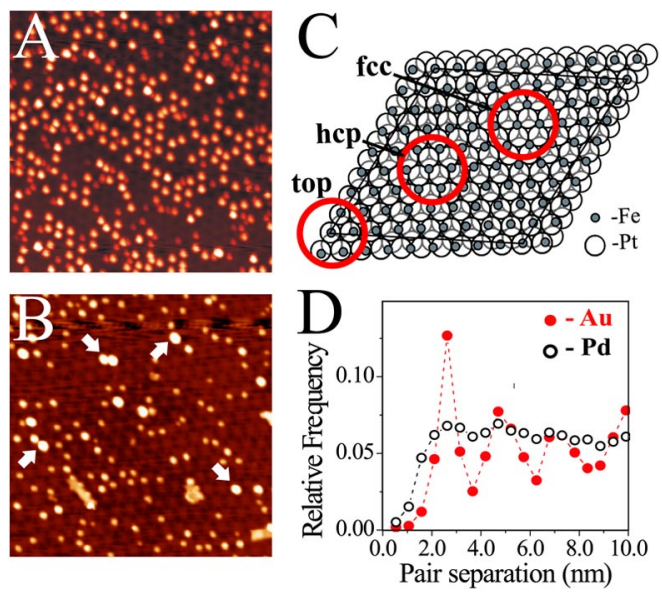

FIG. 1 (color online). STM topographic images of (a) Au and (b) $\mathrm{Pd}$ adatoms on $\mathrm{FeO} / \mathrm{Pt}(111)\left(U_{\text {Sample }}=250 \mathrm{mV}, 50 \times\right.$ $50 \mathrm{~nm}^{2}$ ). The coverage amounts to 0.75 and 0.45 atoms per $\mathrm{FeO}$ Moiré unit cell in (a) and (b), respectively. Arrows in (b) mark small Pd clusters. (c) Graphical representation of the Moiré cell with indication of the binding sites. (d) Radial pair distribution function for $\mathrm{Au}$ and $\mathrm{Pd}$ atoms on $\mathrm{FeO} / \mathrm{Pt}(111)$, as deduced from evaluating the distance of atom pairs from several STM topographic images. images [Figs. 1(a) and 1(b)], but becomes apparent in radial distribution functions which plot the probability to find a neighboring metal atom in a certain distance from a first one [Fig. 1(d)]. In the case of Au, this distribution function is governed by well pronounced peaks at (26 \pm $0.5),(48 \pm 1)$, and $(75 \pm 2) \AA$, fully compatible with the periodicity of the Moiré cell. For Pd, the pair distribution function shows an initial increase to a broad maximum at $(26 \pm 3) \AA$ but is nearly flat for larger pair separations. This emphasizes the weak ordering of Pd that has already been deduced from the topographic images [Fig. 1(b)]. The different spatial arrangements of $\mathrm{Au}$ and $\mathrm{Pd}$ adatoms reflect, in addition, the different aggregation behavior of both species. While gold adatoms adsorb principally as monomers, Pd tends to form small clusters, which efficiently hinders any densification of the adatom lattice. The self organization of Au-atoms has already been pointed out and ascribed to a modulation of the surface potential of the polar FeO film and a repulsion between the adatoms [20]. However, neither the nature of the interaction nor a possible charging of the adatoms could be clarified from those experiments [20]. Here, we demonstrate that the ordering is connected to the occurrence of a charge transfer from the atoms to the substrate.

For the calculations, we used the DFT $+\mathrm{U}$ approach $(U=4, J=1)$, as formulated by Dudarev [21] and implemented in the VASP code [22,23], with the generalized gradient approximation (GGA) and the Perdew-Wang 91 (PW91) functional [24] (plane wave basis set with kinetic energy cutoff of $400 \mathrm{eV}$, and projector augmented wave method $[25,26])$. The $\mathrm{FeO} / \mathrm{Pt}(111)$ interface is modeled by a non-pseudomorphic cell that corresponds to a superposition of $(\sqrt{ } 7 \times \sqrt{ } 7) R 19^{\circ}-\mathrm{FeO}(111)$ and $(3 \times 3)-\mathrm{Pt}(111)$ structures [19]. The computational study implies an analysis of the preferential Au and Pd adsorption sites, considering O-top, Fe-top, bridge, and hollow site in the top, hcp, and fcc domains of the Moiré unit cell. The calculated adsorption site for $\mathrm{Au}$ that best matches the experimental evidence is the $\mathrm{O}$-top one. The corresponding adsorption energy of about $0.6 \mathrm{eV}$ is $0.2 \mathrm{eV}$ larger than that of the Fetop sites, while bridge and hollow sites do not produce stable adsorption geometries. By comparing O-top sites in different domains of the Moiré pattern, we found a preference of about $0.2 \mathrm{eV}$ for the hcp one, in agreement with the experimental results. At this site, the Au-O distance is $2.1 \AA$, and the Au adsorption induces a considerable local increase of the rumpling of the $\mathrm{FeO}$ layer $(20 \%)$ and a small reduction of the FeO-Pt interface distance (1\%). This strong polaronic distortion is a signature of the charged nature of the adatom [3]. For the sake of completeness, it should be mentioned that the calculations predict another, qualitatively different adsorption configuration, where the $\mathrm{Au}$ adatom binds directly to a $\mathrm{Fe}$ ion that has relaxed above the oxygen layer, reversing locally the rumpling of the $\mathrm{FeO}$ film [27]. We attribute the absence of such configurations 
in the experiments to a kinetic barrier to reverse the local rumpling. A full discussion of this specific configuration will be presented elsewhere [27].

The local density of states (LDOS) projected on an Au in an O-top site is reported in Fig. 2(a). Because of the wellknown limitations of DFT methods to describe empty states, and considering the delicate choice of the $\mathrm{U}$ parameter in DFT $+\mathrm{U}$ calculations, the comparison with $d I / d V$ curves is by necessity qualitative. The $6 s$ state is clearly above the Fermi level $\left(E_{F}\right)$, providing a strong indication that one electron has been transferred from the $\mathrm{Au} 6 s$ level to the substrate. This is also consistent with the calculated local decrease of $\Phi$ by $0.9 \mathrm{eV}$. The reduction is partly counterbalanced by the Au-induced $\mathrm{FeO}$ rumpling which acts to increase $\Phi$. The presence of an unoccupied Au $6 s$ orbital is also suggested by $d I / d V$ spectroscopy, which provides a measure of the LDOS according to the Tersoff-Hamann model. In spectra taken above an Au atom located in an hcp domain, a pronounced peak is revealed at $500 \mathrm{meV}$ above $E_{F}$, in good agreement to the calculated $6 \mathrm{~s}$ position of $300 \mathrm{meV}$ (Fig. 3). Experimental evidence for the Au $6 s$ character of the state in the $d I / d V$ spectra is revealed by dosing the surface to $\mathrm{CO}$. In AuCO complexes formed upon $\mathrm{CO}$ adsorption, the resonance is quenched and the carbonyl species therefore appear with smaller topographic height than bare $\mathrm{Au}$ atoms [Fig. 3(a)]. Indeed, the DFT calculations show that the $6 s$ state, hybridized with the Au $5 d_{z}^{2}$ orbital, interacts with the $\mathrm{CO} 5 \sigma$ state and splits into a bonding state at $-3.5 \mathrm{eV}$ below $E_{F}$ and an antibonding one at $+3.5 \mathrm{eV}$ [Fig. 2(b)]. This explains the experimentally observed CO-induced shift of the $500 \mathrm{meV}$ peak out of the accessible spectral range for $d I / d V$ spectroscopy. At the same time, the $5 d_{x z}$ and $5 d_{y z}$ components strongly mix with the $\mathrm{CO} \pi^{*}$ states to give an additional empty resonance at $\approx 1 \mathrm{eV}$ above $E_{F}$ in the LDOS [Fig. 2(b)]. This peak is not seen in the $d I / d V$ spectra because of small overlap of the tip wave function with localized $d$ states.

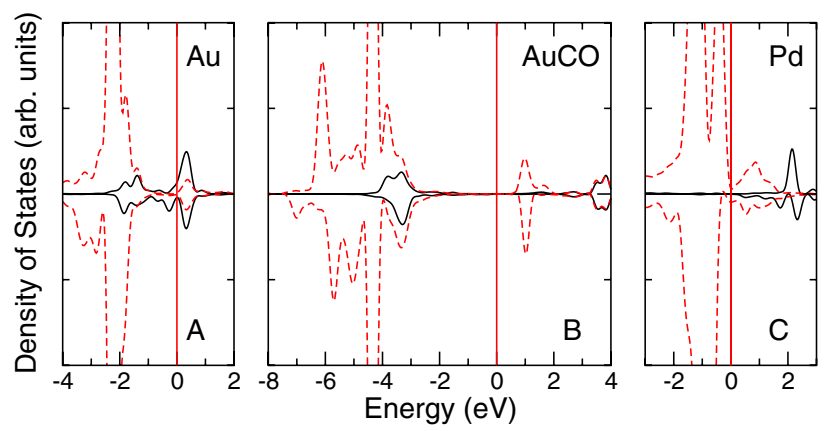

FIG. 2 (color online). Density of states projected on transition metal adatom $d$ (dashed lines) and $s$ (full lines) states, for (a) Au adatoms at $O_{\text {hcp }}$ sites, (b) AuCO complex at $O_{\text {hcp }}$ sites, and (c) Pd atoms in hollow site of the fcc domain. The zero energy is aligned with the Fermi level (dotted vertical lines).
Although some care is necessary when probing the charge state of a metal atom by $\mathrm{CO}$ adsorption [28], the computed properties of the AuCO complex are clearly indicative of adsorption at a positively charged species. In contrast to neutral or negatively charged AuCO complexes, the calculations show that $\mathrm{CO}$ is strongly bound to $\mathrm{Au}$ on $\mathrm{FeO} / \mathrm{Pt}(111)$ by $2.3 \mathrm{eV}$, and the AuCO complex is linear and normal to the surface with short $r(\mathrm{Au}-\mathrm{CO})=$ 1.9 A. Such a strong AuCO bond is typical of gold cations: the measured bond strength in gas-phase $\mathrm{Au}^{+}-\mathrm{CO}$ is $2.08 \pm 0.15 \mathrm{eV}[29]$.

In contrast to $\mathrm{Au}$, no preferential adsorption site or domain could be identified for Pd, and all three stacking regions of the $\mathrm{FeO}$ Moire cell are occupied with similar probability, Fig. 1(b). This is consistent with the DFT results which show that the Pd binding energies are rather similar for the different adsorption sites and depend only moderately on the particular domain. In general, bridge sites where Pd adatoms are coordinated to two oxygens are preferred over Fe-top and O-top sites. The most stable adsorption site is a threefold coordinated hollow site in the fcc Moiré domain, located directly atop of a substrate $\mathrm{Pt}$ atom. The computed adsorption energies are always larger than for $\mathrm{Au}$, and range from $1.3 \mathrm{eV}$ for bridge to $1.5 \mathrm{eV}$ for the hollow site. The adsorption induces a modest increase of the $\mathrm{FeO}$ rumpling (3\%) and a negligible modification of the FeO-Pt interface separation.

The calculated LDOS, Fig. 2(c), shows that the Pd $4 d$ states are between $-2 \mathrm{eV}$ and $E_{F}$, with a small component crossing the Fermi level. This is indicative of a bonding with the surface oxygen which leads to a partial oxidation of the adatom and results in a reduction of the calculated local work function by $0.9 \mathrm{eV}$. Notice that this value is the same as computed for the Au cation only because in the $\mathrm{Au}$ case, the large polaronic distortion acts to partially cancel the effect. In the computed LDOS, the diffuse Pd $5 s$ state is located $2 \mathrm{eV}$ above the Fermi level. Experimentally, the Pd atoms in different adsorption environments show no $d I / d V$ features in a spectral window between -1.5 and $+1.5 \mathrm{~V}$ (not shown). Larger bias voltages cannot be applied as they
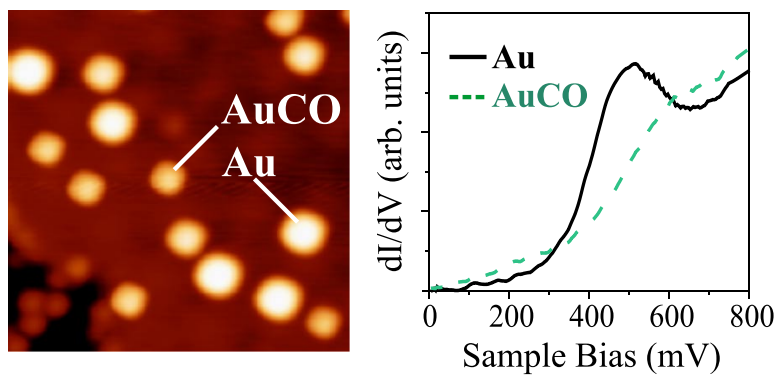

FIG. 3 (color online). STM image and $d I / d V$ spectra of Au and $\mathrm{AuCO}$ complexes on $\mathrm{FeO} / \mathrm{Pt}(111)\left(U_{\text {Sample }}=650 \mathrm{mV}\right.$, $\left.12 \times 12 \mathrm{~nm}^{2}\right)$. The setpoint for spectroscopy was fixed with $800 \mathrm{mV}$ and $0.5 \mathrm{nA}$. 
stimulate diffusion and desorption of the adatoms. The absence of conductance peaks is consistent with the calculated LDOS, as the $\mathrm{Pd} 5 s$ state is outside the spectral range for spectroscopy and, as mentioned above, the $d$ states are usually too localized to induce substantial overlap with the tip wave functions [Fig. 2(c)]. Also, CO adsorption has no measurable effect on the apparent height or electronic structure of $\mathrm{Pd}$ adatoms on $\mathrm{FeO} / \mathrm{Pt}(111)$.

The previous discussion shows that upon adsorption on the Pt-supported $\mathrm{FeO}(111)$ oxide film, Au loses its $6 \mathrm{~s}$ electron and forms a cationic species, while $\mathrm{Pd}$ is only partly oxidized and remains formally "neutral." One important difference between the two metal species is that Pd atoms have a clear tendency to form covalent bonds with substrate oxygen ions, which leads to larger adsorption energies, and preferred adsorption on sites where the coordination is high. Conversely, the adsorption of Au adatoms is weaker and only atop adsorption geometries are stable. The contribution of covalent bonding is considerably smaller, and electrostatic effects play a more important role. Unfortunately, due to the very different nature of the electronic states involved and the known deficiencies of charge evaluation methods, we are unable to provide a quantification of the different charge state in the two cases.

In conclusion, we have presented a study on $\mathrm{Au}$ and $\mathrm{Pd}$ adatoms deposited on Pt-supported $\mathrm{FeO}(111)$ films. $\mathrm{Au}$ atoms form a regular array while Pd atoms are randomly distributed at the surface. The combined experimental and theoretical approaches provide an explanation for this behavior: whereas Pd remains neutral upon adsorption, $\mathrm{Au}$ becomes cationic, thus stimulating repulsion and selfordering of the adatoms. A similar effect has been observed already for the same atoms on $\mathrm{MgO}$ ultrathin films on $\operatorname{Ag}(001)$, but there, the charge transfer occurs in the opposite direction [6]. The formation of $\mathrm{Au}$ anions on $\mathrm{MgO} / \mathrm{Ag}(001)$ versus cations on $\mathrm{FeO} / \mathrm{Pt}(111)$ manifests the work function influence on the charge flow in both thin film systems. The magnesia layer reduces the $\operatorname{Ag}(001)$ work function [30], supporting electron transfer into the half-filled $\mathrm{Au} 6 s$ orbital. The $\mathrm{FeO} / \mathrm{Pt}(111)$ system, on the other hand, has an exceptionally high work function. As a consequence, the Au $6 s$ level is pushed above the Fermi energy and empties into the support in the latter case. Because of the predominately covalent bonding, Pd atoms are less affected by the high work function and become only partly oxidized. This study shows for the first time the possibility to induce a charge transfers in both directions for $\mathrm{Au}$ atoms adsorbed on different oxide thin films, thus providing another step in the direction of designing metal/ oxide interfaces with specific and uncommon properties.
We are grateful to C. Noguera for fruitful discussions. We thank the COST Action D41 "Inorganic oxides: surfaces and interfaces." Part of the computing time was provided by the Barcelona Supercomputing CenterCentro Nacional de Supercomputación (BSC-CNS).

*Corresponding authors: gianfranco.pacchioni@unimib.it; nilius@ fhi-berlin.mpg.de

[1] H. J. Freund, Surf. Sci. 601, 1438 (2007).

[2] G. Pacchioni, L. Giordano, and M. Baistrocchi, Phys. Rev. Lett. 94, 226104 (2005).

[3] L. Giordano and G. Pacchioni, Phys. Chem. Chem. Phys. 8, 3335 (2006).

[4] D. Ricci et al., Phys. Rev. Lett. 97, 036106 (2006).

[5] K. Honkala and H. Hakkinen, J. Phys. Chem. C 111, 4319 (2007).

[6] M. Sterrer et al., Phys. Rev. Lett. 98, 096107 (2007).

[7] M. Sterrer et al., Phys. Rev. Lett. 98, 206103 (2007).

[8] M. Kulawik, N. Nilius, and H.-J. Freund, Phys. Rev. Lett. 96, 036103 (2006).

[9] N. Nilius et al., Phys. Rev. Lett. 100, 096802 (2008).

[10] U. Martinez, L. Giordano, and G. Pacchioni, J. Chem. Phys. 128, 164707 (2008).

[11] W. Zhao et al., Phys. Rev. B 62, 7527 (2000).

[12] J. Goniakowski, F. Finocchi, and C. Noguera, Rep. Prog. Phys. 71, 016501 (2008).

[13] C. Noguera and J. Goniakowski, J. Phys. Condens. Matter 20, 264003 (2008).

[14] J. Goniakowski, C. Noguera, and L. Giordano, Phys. Rev. Lett. 98, 205701 (2007).

[15] G. H. Vurens, M. Salmeron, and G. A. Somorjai, Surf. Sci. 201, 129 (1988).

[16] Y. J. Kim et al., Phys. Rev. B 55, R13448 (1997).

[17] M. Ritter, W. Ranke, and W. Weiss, Phys. Rev. B 57, 7240 (1998).

[18] E. D. L. Rienks et al., Phys. Rev. B 71, 241404 (2005).

[19] L. Giordano et al., Phys. Rev. B 76, 075416 (2007).

[20] N. Nilius et al., Phys. Rev. Lett. 95, 066101 (2005).

[21] S. L. Dudarev et al., Phys. Rev. B 57, 1505 (1998).

[22] G. Kresse and J. Hafner, Phys. Rev. B 47, R558 (1993).

[23] G. Kresse and J. Furthmüller, Phys. Rev. B 54, 11169 (1996).

[24] J. P. Perdew et al., Phys. Rev. B 46, 6671 (1992).

[25] P. E. Blöchl, Phys. Rev. B 50, 17953 (1994).

[26] O. Bengone et al., Phys. Rev. B 62, 16392 (2000).

[27] L. Giordano et al. (to be published).

[28] M. Sterrer et al., Angew. Chem. Int. Ed. Engl., Suppl. 45, 2633 (2006).

[29] M. Neumaier et al., J. Chem. Phys. 122, 104702 (2005).

[30] L. Giordano, F. Cinquini, and G. Pacchioni, Phys. Rev. B 73, 045414 (2006). 